\title{
Hard $x$-ray single-shot spectrometer at the European X-ray Free-Electron Laser
}

Cite as: Rev. Sci. Instrum. 91, 103101 (2020); https://doi.org/10.1063/5.0019935

Submitted: 26 June 2020 . Accepted: 12 September 2020 . Published Online: 01 October 2020

(iD) Naresh Kujala, Wolfgang Freund, Jia Liu, Andreas Koch, Torben Falk, Marc Planas, Florian Dietrich, Joakim Laksman, Theophilos Maltezopoulos, Johannes Risch, Fabio Dall'Antonia, and Jan Grünert

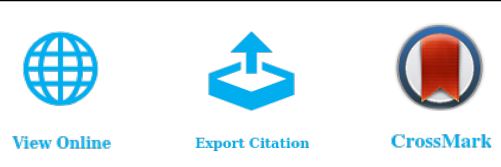

\section{ARTICLES YOU MAY BE INTERESTED IN}

Measurements of ion velocity distributions in a large scale laser-produced plasma

Review of Scientific Instruments 91, 103103 (2020); https://doi.org/10.1063/5.0013447

Monitoring shot-to-shot variations of soft $x$-ray sources using aluminum foils

Review of Scientific Instruments 91, 103001 (2020); https://doi.org/10.1063/5.0021999

An experimental control system for electron spectrometers using Arduino and LabVIEW interfaces

Review of Scientific Instruments 91, 103104 (2020); https://doi.org/10.1063/5.0021229

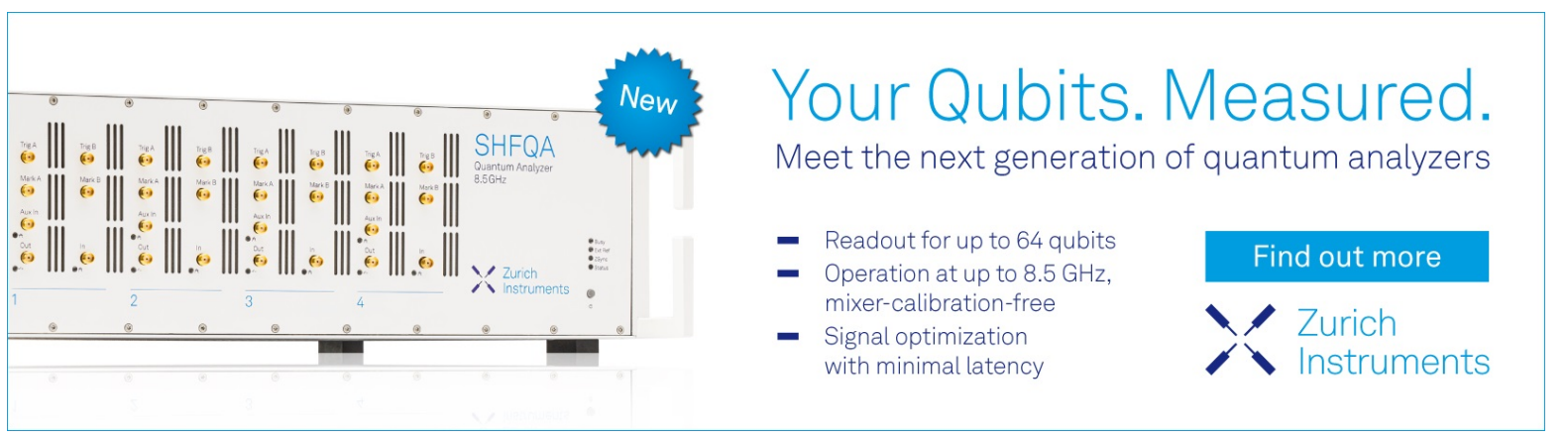




\title{
Hard x-ray single-shot spectrometer at the European X-ray Free-Electron Laser
}

\author{
Cite as: Rev. Sci. Instrum. 91, 103101 (2020); doi: 10.1063/5.0019935 \\ Submitted: 26 June 2020 - Accepted: 12 September 2020 • \\ Published Online: 1 October 2020
}

\begin{abstract}
Naresh Kujala, ${ }^{\text {a) }}$ (Dolfgang Freund, Jia Liu, (D) Andreas Koch, Torben Falk, Marc Planas, Florian Dietrich, Joakim Laksman, (D) Theophilos Maltezopoulos, Johannes Risch, Fabio Dall'Antonia, and Jan Grünert
\end{abstract}

AFFILIATIONS

European X-Ray Free-Electron Laser Facility GmbH, Holzkoppel 4, 22869 Schenefeld, Germany

${ }^{a}$ Author to whom correspondence should be addressed: naresh.kujala@xfel.eu

\begin{abstract}
The European X-ray Free-Electron Laser Facility in Germany delivers X-ray pulses with femtosecond pulse duration at a repetition rate of up to 4.5 MHz. The free-electron laser radiation is created by the self-amplified spontaneous emission (SASE) process, whose stochastic nature gives rise to shot-to-shot fluctuations in most beam properties, including spectrum, pulse energy, spatial profile, wavefront, and temporal profile. Each spectrum consisting of many spikes varies in width and amplitude that appear differently within the envelope of the SASE spectrum. In order to measure and study the SASE spectrum, the HIgh REsolution hard X-ray single-shot (HIREX) spectrometer was installed in the photon tunnel of the SASE1 undulator beamline. It is based on diamond gratings, bent crystals as a dispersive element, and a MHz-repetitionrate strip detector. It covers a photon energy range of $3 \mathrm{keV}-25 \mathrm{keV}$ and a bandwidth of $0.5 \%$ of the SASE beam. The SASE spikes are resolved with $0.15 \mathrm{eV}$ separation using the Si 440 reflection, providing a resolving power of 60000 at a photon energy of $9.3 \mathrm{keV}$. The measured SASE bandwidth is $25 \mathrm{eV}$. In this paper, we discuss the design specifications, installation, and commissioning of the HIREX spectrometer. The spectral results using $\mathrm{Si}(110), \mathrm{Si}$ (111), and C (110) crystals are presented.
\end{abstract}

Published under license by AIP Publishing. https://doi.org/10.1063/5.0019935

\section{INTRODUCTION}

The European X-ray Free-Electron Laser (EuXFEL) is a newly built user facility that started user operation in fall 2017 in the metropolitan area of Hamburg, Germany. ${ }^{1-3}$ The EuXFEL linear accelerator is based on superconducting technology with the capability to accelerate electron bunches within $600 \mu$ s long radiofrequency pulses with an electron bunch charge that varies from $20 \mathrm{pC}$ to $1 \mathrm{nC}$. The $\mathrm{x}$-ray free-electron laser (FEL) is based on the principle of self-amplified spontaneous emission (SASE). ${ }^{4-7} \mathrm{Up}$ to 2700 pulses per train are delivered at a $10 \mathrm{~Hz}$ repetition rate. The pulses in a train are separated by at least $220 \mathrm{~ns}$, and the trains are separated by $100 \mathrm{~ms}$. The pulse structure pattern of the EuXFEL is shown in Fig. 1. This provides the users with ultrashort (femtosecond) and intense (gigawatt) transversely coherent $\mathrm{x}$-ray laser beams in the soft to hard $\mathrm{x}$-ray domain at a $\mathrm{MHz}$ repetition rate. ${ }^{8,9}$ The unique time structure provides not only very high peak brilliance but also the highest average brilliance of all the x-ray FEL facilities worldwide. ${ }^{10-13}$ There are in total three SASE undulators that can produce $\mathrm{x}$-ray FEL radiation: the two hard $\mathrm{x}$-ray undulators, SASE1 and SASE2, generate hard $\mathrm{x}$-ray radiation from 3 to $25 \mathrm{keV}$, while the third undulator, SASE3, provides soft $\mathrm{x}$ rays up to $3 \mathrm{keV}$. In May 2017, the first lasing was detected and the SASE1 undulator beamline was commissioned with the FEL beam. ${ }^{14}$ The X-ray Photon Diagnostics group at the EuXFEL provides photon beam information on set up and operation of the accelerator, undulator, and x-ray optics, as well as diagnostic information to users and instrument end-station. ${ }^{15-18}$

SASE $x$-ray FEL radiation is, by nature, stochastic and, therefore, produces not only strong pulse-to-pulse intensity fluctuations but also strongly fluctuating spectral features. While the mean photon energy per pulse is correlated with the energy of the electron bunch, the details of an individual spectrum, consisting of many spectral modes, cannot be predicted due to its chaotic nature. Inside the envelope of the SASE beam, a characteristic pattern of spectral spikes is present, depending on the pulse duration and the fine structure of micro-bunching that develops in the electron beam as it passes through the undulator section of the beamline. Each singleshot spectrum is different and consists of many spikes with varying width and amplitude. The shot-to-shot fluctuations in the spectrum 


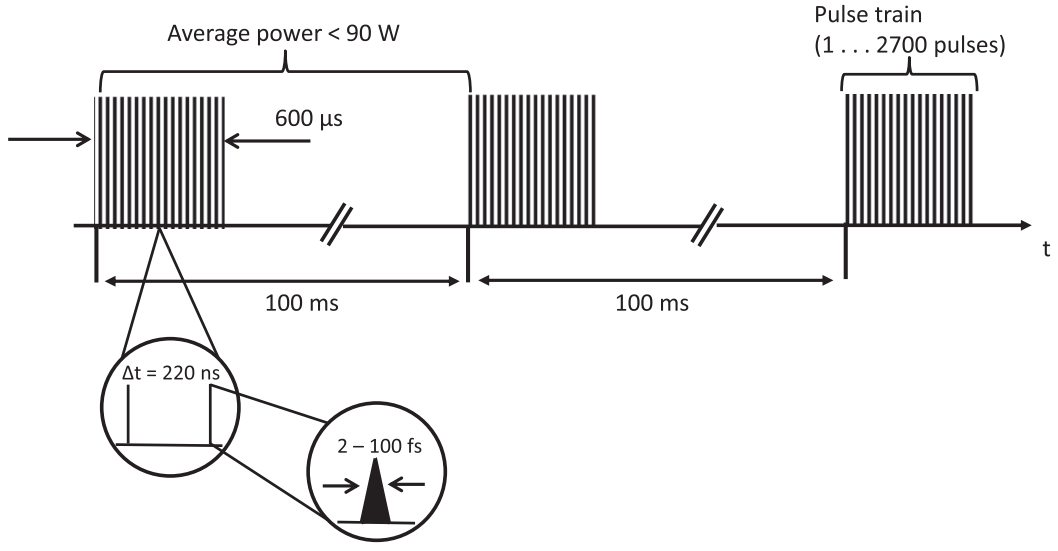

FIG. 1. The pulse structure of the EuXFEL. The pulse trains arrive at a $10 \mathrm{~Hz}$, and the pulses within each train are separated by $220 \mathrm{~ns}(4.5 \mathrm{MHz}$ intra-bunch-train repetition rate).

are due to the SASE process, which was explained by theory and simulations. ${ }^{19}$

Therefore, the EuXFEL has installed the HIgh REsolution Hard $\mathrm{X}$-ray single-shot (HIREX) spectrometer to measure the spectrum of individual photon pulses and to provide this information to the Femtosecond X-ray Experiments (FXE) instrument and the Single Particles, Clusters, and Biomolecules/Serial Femtosecond Crystallography (SPB/SFX) instrument as a means to improve data analysis. It is also important to know the spectrum of the source, on a shot-to-shot basis, for the optimization of the accelerator operation parameters, for instance, in order to stabilize the FEL radiation output. Due to the shot-to-shot fluctuations, the spectral analysis must be performed upstream of the experiment stations on the very same pulse used for the experimental sample interaction, which implies that the spectral monitor must be non-invasive. Ideally, the monitor system should cover the entire hard x-ray photon energy range of $3 \mathrm{keV}-25 \mathrm{keV}$, as planned for the SASE1 undulator operation, and it should operate at an intra-bunch train pulse frequency of up to 4.5 MHz. The HIREX spectrometer is installed in the photon tunnel XTD9 for the SASE1 beamline at $2798.30 \mathrm{~m}$ from the injector gun location and $360.30 \mathrm{~m}$ away from the undulator source point. The tunnel layout and the location of the HIREX are shown in Fig. 2. In this paper, we discuss the design specifications, installation, and commissioning of the HIREX spectrometer.

\section{DESIGN SPECIFICATIONS AND IMPLEMENTATION}

Several techniques have been implemented at different $\mathrm{x}$-ray FEL facilities around the world to measure the hard x-ray singleshot spectrum. ${ }^{20-23}$ The conceptual design and simulation of the different crystal reflections and bending radius selections have been performed using the ray-tracing tool RAY. ${ }^{24,25}$ The principle of the bent crystal spectrometer was first implemented at the Linac Coherent Light Source (LCLS) in the USA, and its working principle is described in Ref. 26. The principle of the grating-based spectrometer has been described by the Paul Scherrer Institute (PSI) in Switzerland. ${ }^{27-29}$ At EuXFEL, we have implemented the HIREX spectrometer based on a diffraction grating to split off a small fraction of the photon beam, a bent crystal as a dispersive element, and a $\mathrm{MHz}$ repetition rate $1 \mathrm{D}$ strip detector. The first-order diffracted beam is used for the spectral measurement. Thus, the HIREX spectrometer is capable of working with $\mathrm{X}$-ray FEL pulses at a $\mathrm{MHz}$ repetition rate that is not available at other facilities, demonstrating the uniqueness of the EuXFEL Facility. The schematic of the HIREX spectrometer is shown in Fig. 3. The technical design specifications are as follows: (a) it covers hard $\mathrm{x}$-ray photon energies in the range of $3 \mathrm{keV}-25 \mathrm{keV}$; (b) it has a resolving power of 40000 (e.g., $0.2 \mathrm{eV}$ at $8 \mathrm{keV})$; (c) it covers up to $1 \%$ of the FEL bandwidth; and (d) it is minimally invasive ( $95 \%$ of the beam energy is transmitted).

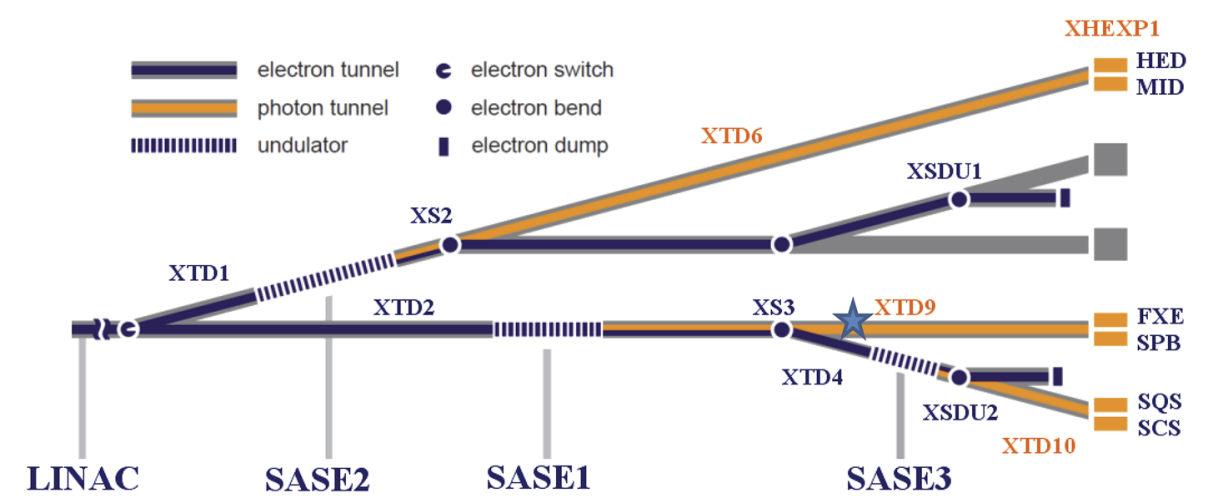

FIG. 2. Schematic of the EuXFEL tunnel layout. The star indicates the location of the HIREX spectrometer in the SASE1 photon tunnel XTD9, which provides spectral information to the Femtosecond X-ray Experiments (FXE) instrument and the Single Particles, Clusters, and Biomolecules/Serial Femtosecond Crystallography (SPB/SFX) instrument. 


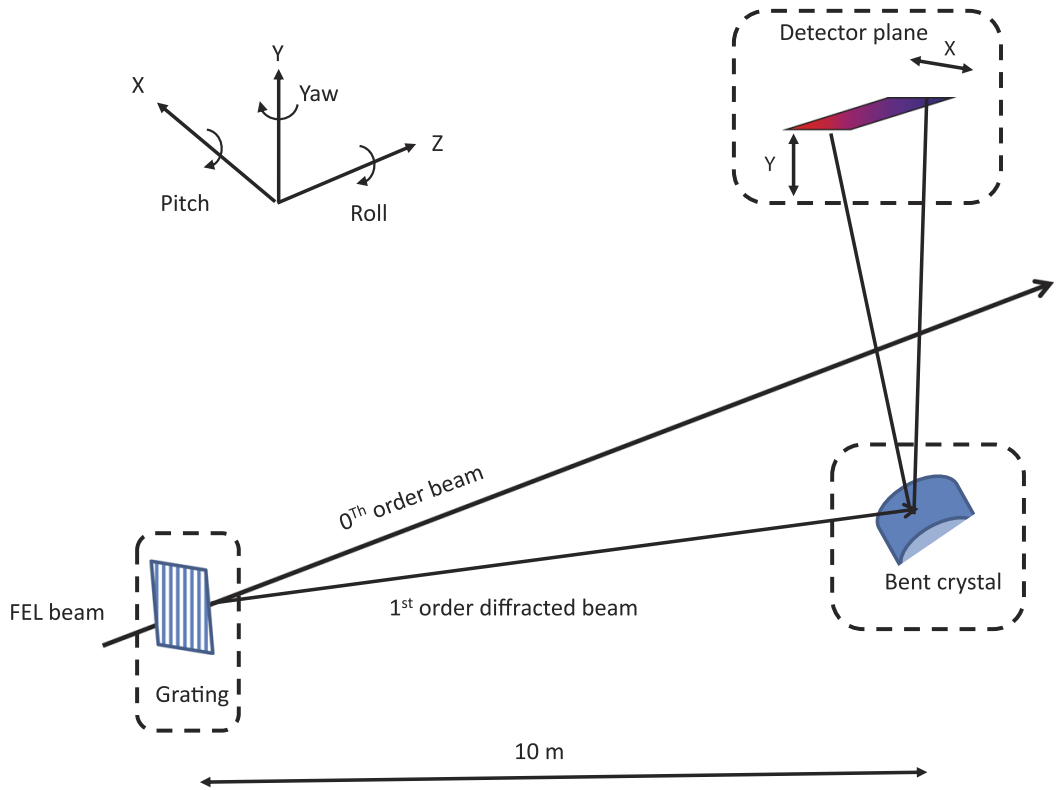

FIG. 3. Schematic of the HIREX spectrometer. The distance between the linear transmissive diamond grating and the bent crystal is $10 \mathrm{~m}$. The detector is $1 \mathrm{~m}$ away from the bent crystal. The grating lines were oriented in the vertical direction normal to the FEL beam so that the diffracted beams were deflected in the horizontal plane.

The energy resolution at the detector plane is calculated by ${ }^{26}$

$$
\frac{\Delta E}{E}=\frac{\Delta x}{2 \tan \theta_{B}\left(\frac{R \sin \theta_{B}}{2}+L\right)},
$$

where $L$ is the distance between crystal and detector, $\Delta x$ is the detector pixel size, and $\theta_{B}$ is the Bragg angle. $R$ is the radius of curvature of the bent crystal.

The spectral range covered by the bent crystal can be calculated by ${ }^{26}$

$$
\frac{\delta E}{E}=\left(\frac{H}{R \sin \theta_{B}}\right) \cot \theta_{B},
$$

where $H$ is the beam size and $R$ is the bending radius of the crystal. For the HIREX spectrometer, the spectral range is directly proportional to the beam size in the dispersion plane and is inversely proportional to the bending radius with an assumption of homogenous spectral distribution across the entire beam profile.

A large number of photons arrive less than $100 \mathrm{fs}$. The average power during a train of $\mathrm{x}$-ray pulses can be as high as several $\mathrm{kW}$, depending on the energy per pulse and the number of pulses per train. Diamond is chosen for grating fabrication due to its high thermal conductivity $(2052 \mathrm{~W} / \mathrm{mK}$ at $300 \mathrm{~K})$, low absorption for hard $\mathrm{x}$ rays, and the higher single-shot damage threshold as compared to silicon. The FEL beam, after passing the gratings, is split into different orders, and the first order diffracted beam, which contains about two orders of magnitude less power, is used for measuring the $\mathrm{x}$-ray spectrum. A CAD model of the HIREX spectrometer of SASE1 is shown in Fig. 4. A photograph of the HIREX installed in the photon tunnel XTD9 is shown in Fig. 5. The mechanical and vacuum system of HIREX is designed by the Axilon AG Hürth, Germany (www.axilon.de)

The CVD diamond gratings with pitches of $200 \mathrm{~nm}$ and $150 \mathrm{~nm}$ were fabricated at the Laboratory for Micro- and Nanotechnology at PSI. The base material for the grating fabrication, CVD diamond membranes on silicon support frames, was purchased from Diamond Materials GmbH, Freiburg, Germany. The membrane has a diameter of $5 \mathrm{~mm}$ and a thickness of $10 \mu \mathrm{m}$, and it is supported by a $500 \mu \mathrm{m}$ thick silicon frame of $10 \times 10 \mathrm{~mm}^{2}$. The grating lines were produced with a line depth of $1 \mu \mathrm{m}$ over a large area of $2 \times 2 \mathrm{~mm}^{2}$, which is large enough to make alignment simple and to accommodate the different beam sizes at different photon energies of the EuXFEL. The details of the fabrication process of the grating structure were already reported in Ref. 30 . The grating manipulator CAD drawing in Fig. 6 shows the slots for mounting the $200 \mathrm{~nm}$ and $150 \mathrm{~nm}$ pitch gratings (one additional $200 \mathrm{~nm}$ pitch grating is mounted as a spare). The photograph (inset) in this figure shows the CVD diamond linear transmissive grating on the grating holder frame. For good heat conduction, we have mounted an indium foil at the interface between the grating and the holder. In addition to the gratings on the manipulator, there is also a Ce:YAG screen with 100 $\mu \mathrm{m}$ thickness for imaging the FEL beam. The grating manipulator rod is mounted on a linear stage that allows motion in the $\mathrm{X}$ and $\mathrm{Y}$ directions and on a rotation stage that provides rotation with respect to the $\mathrm{X}$-axis, as shown in Fig. 6. For blocking higher-order diffraction from the grating, a boron carbide $\left(\mathrm{B}_{4} \mathrm{C}\right)$ block with an aperture of $4 \mathrm{~mm}$ (horizontal) $\times 10 \mathrm{~mm}$ (vertical) is installed on a horizontal motion stage for insertion at a distance of $1 \mathrm{~m}$ downstream of the gratings. To monitor the temperature, we have installed a PT 100 sensor on the grating and $\mathrm{B}_{4} \mathrm{C}$ manipulator rod. The grating manipulator rod and $\mathrm{B}_{4} \mathrm{C}$ manipulator rod are connected with a copper braid to a water-cooled copper block for heat removal by conduction. In addition to the $\mathrm{B}_{4} \mathrm{C}$ aperture, there are copper and tungsten foils mounted on the manipulator to enable energy calibration of the HIREX spectrometer crystals. All components (excluding the support stands and cables) of the grating unit were assembled under particle-free conditions in a cleanroom of ISO Class 5 level. Prior to the installation of gratings in the grating chamber, the diffraction efficiency and homogeneity of the gratings were measured at the P10 


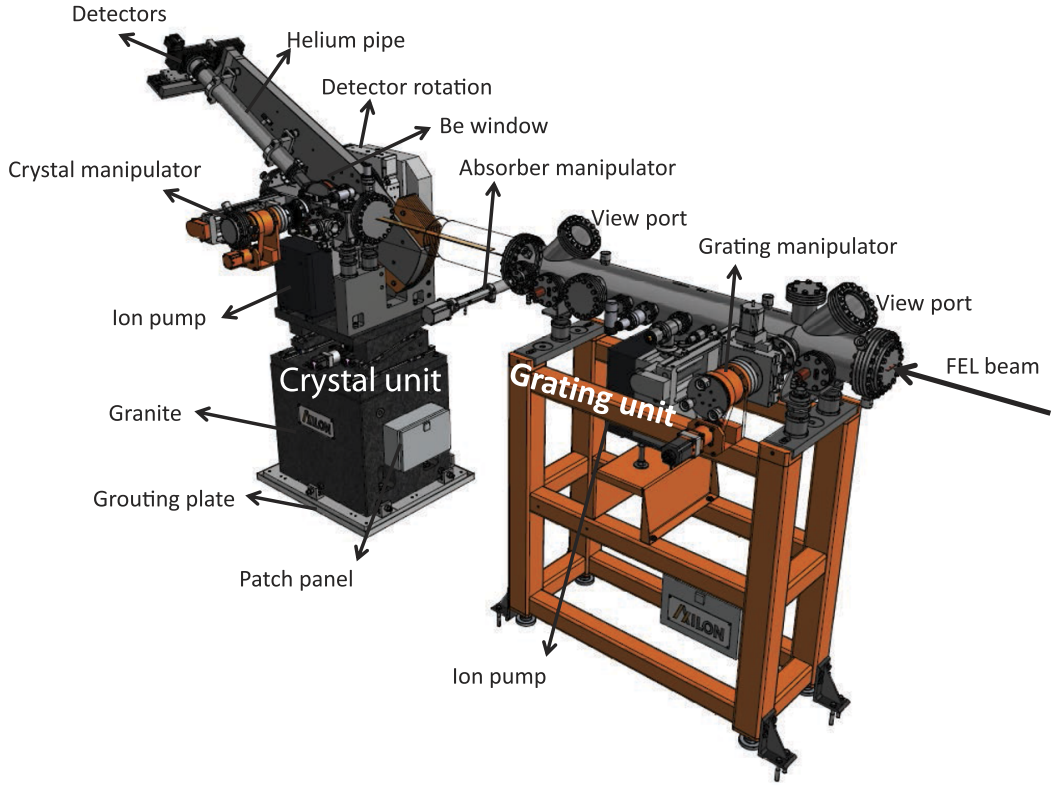

FIG. 4. CAD model of the SASE1 HIREX spectrometer. It consists of a grating unit and a crystal unit. On each unit, an ion pump with a speed of $150 \mathrm{l} / \mathrm{s}$ is used. beamline of PETRA III at DESY Hamburg, Germany. ${ }^{31}$ At a photon energy of $6 \mathrm{keV}, 96 \%$ of the incoming intensity is transmitted in the zeroth order beam, $2 \%$ is absorbed by the diamond membrane, and $2 \%$ is distributed into the diffraction orders. The distance of $10 \mathrm{~m}$ between the gratings and bent crystals allows transverse spatial separation between the zeroth and the first order diffracted beam. The $200 \mathrm{~nm}$ pitch gratings have been commissioned with the FEL beam, and spectral data have been acquired. Figure 7 shows the calculated beam size at the HIREX location in the XTD9 tunnel. ${ }^{3}$

The crystal unit consists of a vacuum chamber, a detector arm, and detectors. The crystal chamber is mounted on linear stages for $\mathrm{X}$ and $\mathrm{Y}$ directions supported on a granite block, and the complete

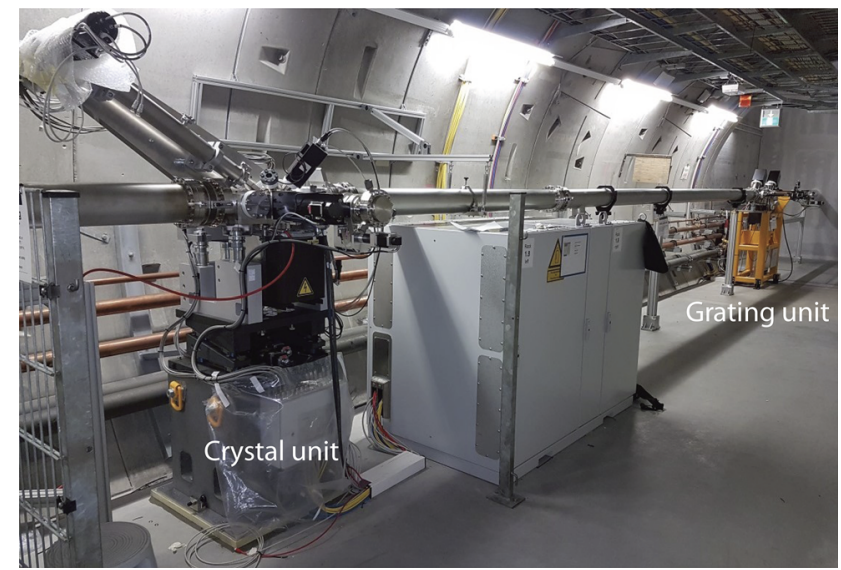

FIG. 5. Photograph of the HIREX spectrometer in the tunnel XTD9 with a safety fence around the crystal unit to protect the beryllium window against damage and to protect workers against the detector arm motion. The FEL beam direction in the image is from right to left. Upstream of the crystal unit is a dedicated electronics rack for motor and vacuum controllers. crystal unit is fixed on an aluminum plate that is grouted with the epoxy of $18 \mathrm{~mm}$ thickness on the tunnel floor. Crystals with a fixed bending radius were installed in the vacuum chamber, and a $250 \mu \mathrm{m}$ thick beryllium foil was brazed on a DN 150 flange (purchased from Materion Company, CA, USA). This beryllium window flange is the interface between vacuum and air, where the FEL beam exits the chamber and propagates toward the detector system. A helium-filled tube is installed between the beryllium window flange and the detector to eliminate absorption and scattering in air. To accommodate the EuXFEL photon energy range of $3 \mathrm{keV}-25 \mathrm{keV}$, we have installed multiple crystals of various orientations and with several bending radii of $150 \mathrm{~mm}, 100 \mathrm{~mm}, 75 \mathrm{~mm}$, and $50 \mathrm{~mm}$. Depending on the machine operation parameters, we choose the

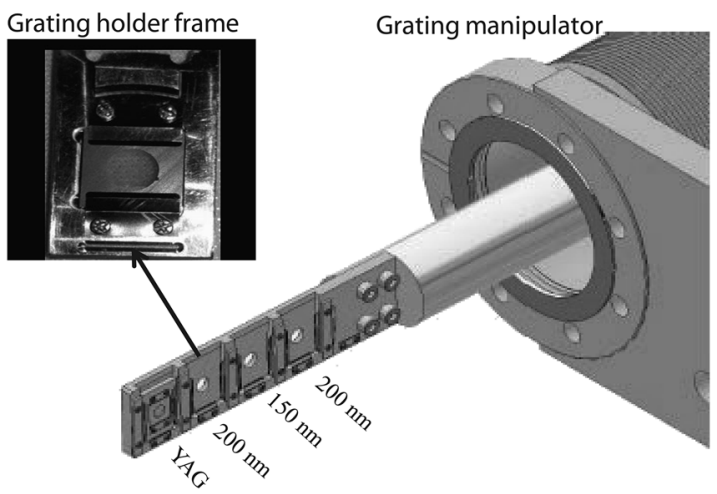

FIG. 6. CAD drawing of the grating manipulator with four slots for mounting the gratings with $150 \mathrm{~nm}$ and $200 \mathrm{~nm}$ pitches (one additional $200 \mathrm{~nm}$ grating mounted as a spare) and one Ce:YAG screen. (Inset) Photograph of the CVD diamond linear transmissive grating on the holder frame. For good heat conduction, we have mounted an indium foil at the interface between the grating and the holder. 


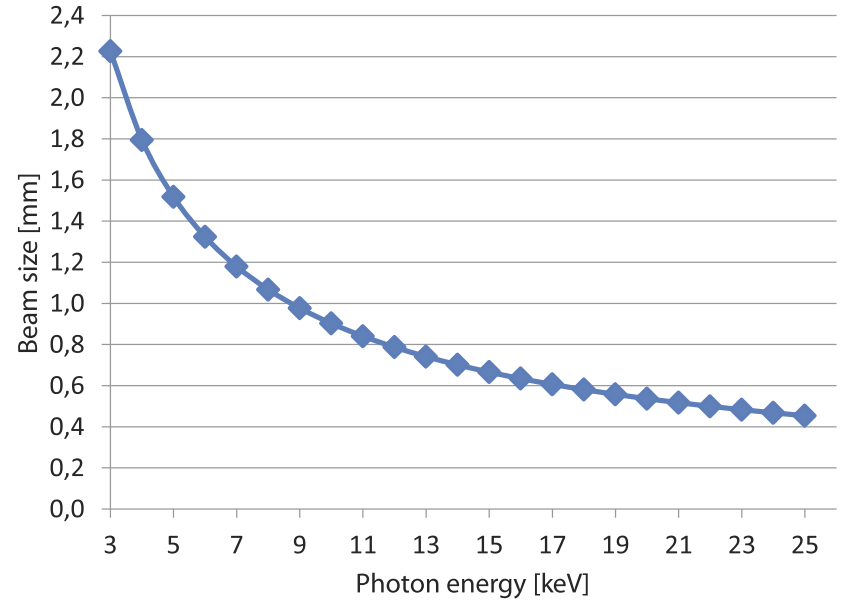

FIG. 7. Beam size (FWHM) at the HIREX location for photon energies of $3 \mathrm{keV}$ $-25 \mathrm{keV}$. The beam size was calculated based on Ref. 32 .

combination of crystal orientation and bending radius for measuring the single-shot spectrum. The crystal chamber was equipped with a crystal holder containing three Si crystals (Norcada Company, Alberta, Canada) of $10 \mu \mathrm{m}$ thickness with 110 and 111 orientation. A Ce:YAG screen is mounted on the same holder for imaging the beam, which is required for the beam-based alignment of the crystals. In order to allow for small bending radii, we have chosen thin silicon crystals with transverse dimensions of $4 \times 20 \mathrm{~mm}^{2}$. They are mounted with a $100 \mu \mathrm{m}$ thick clamp to an aluminum holder with a fixed bending radius, which attaches to the crystal manipulator support rod. The crystal holders are $30 \mathrm{~mm}$ apart in order to pass the zeroth order beam to downstream instruments and at the same time to intercept the first order beam for the spectral measurement. The crystal manipulator rod is connected with a copper braid to a water-cooled copper block, and the temperature is monitored with a PT 100 sensor. In the meantime, we have upgraded one of the crystal positions ( $150 \mathrm{~mm}$ bending radius silicon crystal) to a HPHT bent diamond crystal type IIa (Technological Institute for Superhard and Novel Carbon Materials, Moscow, Russia) with 110 orientation and $125 \mathrm{~mm}$ bending radius. ${ }^{33-36}$ All components (excluding the granite, detectors, and cables) of the crystal chamber are assembled under particle-free conditions in a cleanroom of ISO Class 5 level. Table I lists the types of crystals installed in the crystal chamber. Crystal reflection and orientation is selected based on the photon energy. Figure 8 shows the CAD drawing of the crystal manipulator with photographs (inset) of the silicon crystal holder and the bent diamond crystal holder. The bending radii of the crystals were measured by white-light interferometry using a Wyko NT9100 profilometer (Veeco Instruments Inc.) in the X-ray Optics Laboratory at EuXFEL. The miscut of the Si crystals was measured with a rotating anode $\mathrm{X}$-ray tube source at the Nano Laboratory and Optics Laboratory at DESY. The Si (111) and $\mathrm{Si}$ (110) have a miscut in the dispersion plane of $0.6^{\circ}$ and $0.8^{\circ}$, respectively.

We have installed two detection systems on the crystal unit. These detectors are placed in air on a horizontal linear stage, one detector for train-to-train observations at a low repetition rate of $10 \mathrm{~Hz}$ and the other detector for a high repetition rate to resolve pulses within trains. Figure 9 shows a photograph of the two detectors. The optical detector system for low repetition rates is composed

TABLE I. Crystals mounted in the crystal chamber covering various photon energy ranges. Between $3 \mathrm{keV}$ and $5 \mathrm{keV}$ photon energy, Si (111) crystals will be used.

\begin{tabular}{lccccc}
\hline \hline Bender & Radius $(\mathrm{mm})$ & Bragg angle $(\mathrm{deg})$ & Energy $(\mathrm{keV})$ & Crystals & Crystal reflection \\
\hline 1 & 50 & $37.25-22.8$ & $16-25$ & $\mathrm{Si}(110)$ & 660 \\
2 & 75 & $38.63-21.75$ & $9.5-16$ & $\mathrm{Si}(111)$ & 333 \\
3 & 100 & $40.22-19.80$ & $5-9.5$ & $\mathrm{Si}(110)$ & 220 \\
4 & 125 & $44.6-20.55$ & $7-14$ & $\mathrm{C}(110)$ & 220 \\
5 & Ce:YAG screen & & & \\
\hline \hline
\end{tabular}

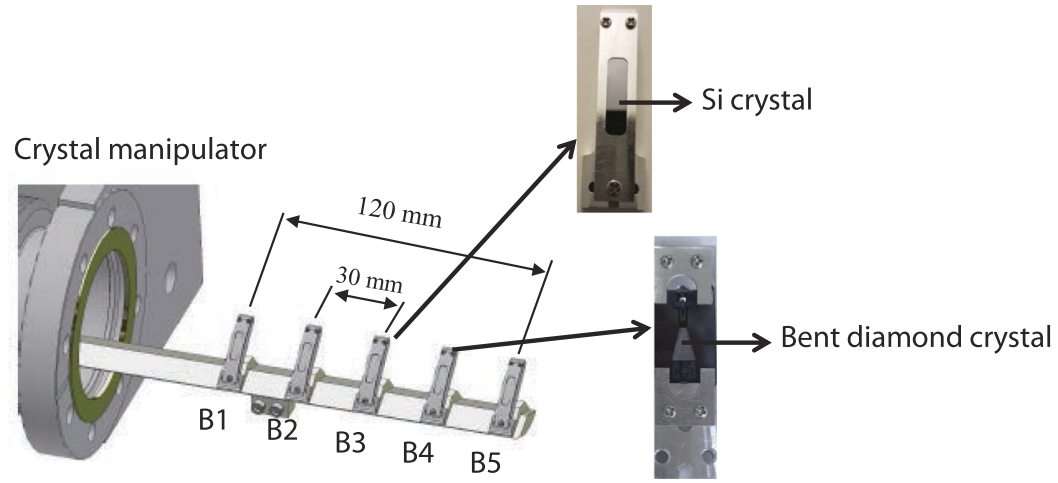

FIG. 8. CAD drawing of the crystal manipulator with photographs (insets) of the $\mathrm{Si}$ and bent diamond crystals clamped on the holder, which, in turn, is mounted on the crystal manipulator support rod. 


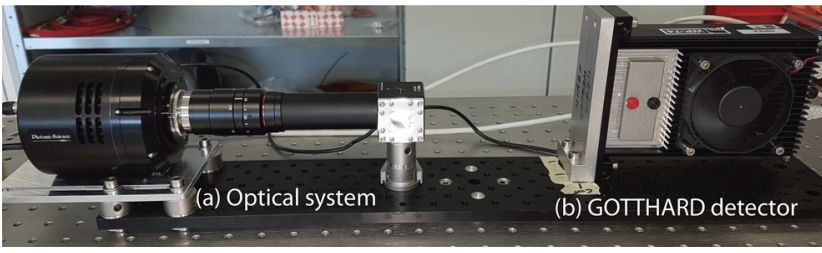

FIG. 9. Photograph of the HIREX spectrometer detector system. (a) The optical system on the left consists of an SCMOS optical camera, optical lenses, and a Ce:YAG screen with a $45^{\circ}$ plane mirror. The YAG screen is covered with a $10 \mu \mathrm{m}$ thick Kapton foil that is coated with Al to avoid stray visible light entering the camera. (b) The detector on the right is a GOTTHARD 1D x-ray detector. Both detector systems are mounted on a breadboard, which, in turn, is mounted on a linear stage for horizontal motion.

of the following parts: (a) sCMOS optical camera, $2 \times 1 \mathrm{k}^{2}$ pixel array with $6.5 \mu \mathrm{m}$ pixel size (Photonic Science and Engineering Ltd., St Leonards, UK), (b) optical objective (Kowa Company, Japan), and (c) $50 \mu \mathrm{m}$ thick Ce:YAG screen (Crytur, Czech Republic) with a $45^{\circ}$ plane mirror. For high repetition rates in the $\mathrm{MHz}$ range, we used a Gain Optimizing microsTrip system witH Analog ReaDout (GOTTHARD) $x$-ray detector with a pixel pitch of $50 \mu \mathrm{m}$. This detector was designed and fabricated at PSI.

The details of the mechanical motion specification for the grating and crystal unit motors are listed in Table II. The motors are controlled via the Beckhoff and ELMO motor controller. The HIREX is located closely upstream of the distribution mirror in the XTD9 tunnel in a particle-free area of the beamline vacuum system. To avoid potential contamination, we keep all actuating motions outside the vacuum system. The in-vacuum rotation and horizontal motion of the gratings and crystals were realized by a magnetic rotary feedthrough and a bellow, respectively. An in-vacuum absolute rotary encoder was utilized.

\section{MEASUREMENTS}

X-ray FEL pulses have many narrow spikes that originate from the stochastic nature of the SASE process, and the spike structure changes shot by shot. The width and number of spikes in the spectrum are related to the x-ray FEL pulse duration and the number of longitudinal modes. The bent crystal disperses the incident $\mathrm{x}$-ray beam since different parts of the incoming beam in the vertical direction have different incident angles. The wavelength that satisfies the Bragg condition will diffract, and thus, the wavelengths in the beam are dispersed along the linear detector, which is placed $1 \mathrm{~m}$ away from the bent crystal in the diffracted beam.

We have commissioned the HIREX spectrometer at different $\mathrm{x}$-ray photon energies. Here, we present results at the photon energy of $9.3 \mathrm{keV}$, which is the preferred photon energy of the EuXFEL machine during startup and tuning. The machine was operated with $14 \mathrm{GeV}$ electron beam energy, $0.25 \mathrm{nC}$ bunch charge, and a repetition rate of $1.1 \mathrm{MHz}$, resulting in $2 \mathrm{~mJ}$ average energy per pulse. The FEL beam size on the HIREX bent crystal at $9.3 \mathrm{keV}$ is $900 \mu \mathrm{m}$ FWHM. Here, we present the results using the 220 and 440 reflections of the Si crystal with a bending radius of $100 \mathrm{~mm}$, using the 330 reflection of the Si crystal with a bending radius of $75 \mathrm{~mm}$, and using the 220 reflection of the bent diamond crystal with a bending radius of $125 \mathrm{~mm}$. The spectral range and resolution of the HIREX spectrometer are determined by the selected Bragg reflection and the associated energy bandwidth, which are determined by Eqs. (1) and (2). We have therefore selected two reflections: one that gives a lower resolution but broader spectral range and the other that allows for a higher resolution with a spectral range sufficiently wide to cover the FWHM spectral bandwidth of the FEL beam. In the single-bunch mode, the spectrum was recorded with a high-resolution 2D sCOMS camera (Photonic Science camera) at $10 \mathrm{~Hz}$ using the Si (220), Si (440), and Si (333) Bragg reflections. In the multiple-bunch mode, the recording of the spectrum from $\mathrm{C}(220)$ in the direct beam and Si (220) in the first order diffracted beam with $200 \mathrm{~nm}$ grating were monitored with a high-speed $1 \mathrm{D} \mathrm{x}$-ray GOTTHARD detector at a repetition rate of $0.5 \mathrm{MHz}$. The $2 \mathrm{D}$ spectral images from the $\mathrm{Si}$ (220) and $\mathrm{Si}$ (440) crystal reflections in the single-bunch mode are shown in Figs. 10(a) and 10(d), respectively. Figures 10(b) and 10(e) show the spectral projections of the $2 \mathrm{D}$ spectrograms normal to the dispersion direction. The Si (220) reflection allows covering the entire SASE spectrum with low resolution and a minimum spike separation of $0.55 \mathrm{eV}$, as shown in Fig. 10(c). The $\mathrm{Si}$ (440) reflection enables high resolution with a minimum spike separation of $0.15 \mathrm{eV}$, as shown in Fig. 10(f). The SASE bandwidth was measured to be $25 \mathrm{eV}$ FWHM.

TABLE II. Specifications for the motors in the grating and crystal unit of the SASE1 HIREX spectrometer, including strokes, resolutions, and encoders.

\begin{tabular}{lcccc}
\hline \hline Axis & Stroke & Resolution & Repeatability & Encoder/resolution \\
\hline Grating X translation & $150 \mathrm{~mm}$ & $2.5 \mu \mathrm{m}$ & $100 \mu \mathrm{m}$ & Linear absolute encoder/50 $\mathrm{nm}$ \\
Grating Y translation & $10 \mathrm{~mm}$ & $1.875 \mu \mathrm{m}$ & $100 \mu \mathrm{m}$ & \\
Grating RX rotation (deg) & 10 & 0.00225 & 0.05 & Incremental/0.001 \\
Absorber X translation & $150 \mathrm{~mm}$ & $50 \mu \mathrm{m}$ & $100 \mu \mathrm{m}$ & Linear absolute encoder/50 nm \\
Crystal/detector X translation & $100 \mathrm{~mm}$ & $0.5 \mu \mathrm{m}$ & $100 \mu \mathrm{m}$ & \\
Crystal/detector Y translation & $10 \mathrm{~mm}$ & $0.5 \mu \mathrm{m}$ & $100 \mu \mathrm{m}$ & \\
Crystal X translation & $150 \mathrm{~mm}$ & $2.5 \mu \mathrm{m}$ & $100 \mu \mathrm{m}$ & \\
Crystal RX rotation (deg) & 45 & 0.00225 & 0.005 & Angular absolute encoder/0.002 \\
Detector RX rotation & 120 & 0.001 & 0.005 & \\
Detector X translation & $295 \mathrm{~mm}$ & $5 \mu \mathrm{m}$ & $100 \mu \mathrm{m}$ & Linear encoder/0.5 $\mu \mathrm{m}$ \\
\hline \hline
\end{tabular}


$\operatorname{Si}(220)$
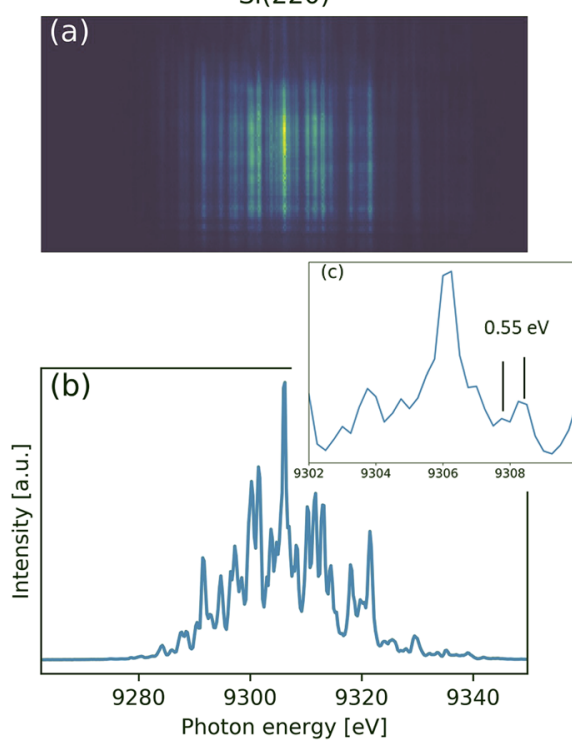

$\operatorname{Si}(440)$
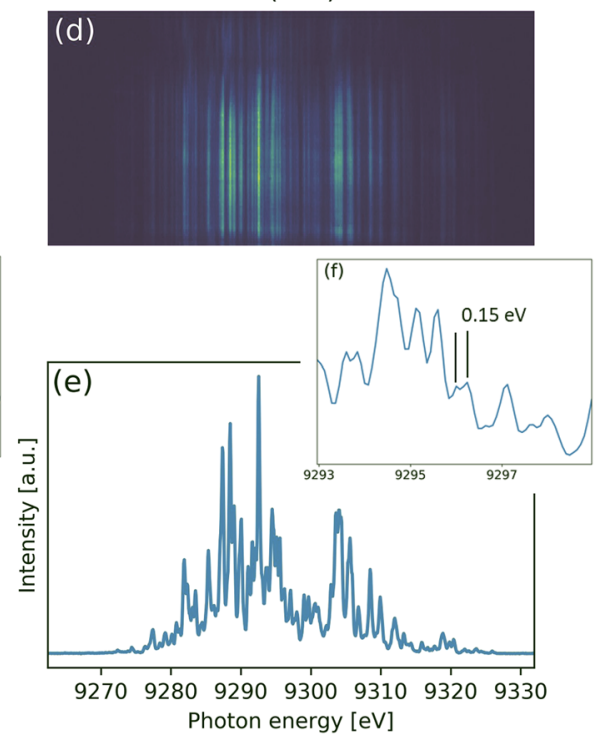

FIG. 10. Single-shot spectra using the $\mathrm{Si}$ crystal with a $100 \mathrm{~mm}$ bending radius in the 220 and 440 reflection, respectively, at $9.3 \mathrm{keV}$. [(a) and (d)] The 2D spectrograms from the optical detector system. $[(b)$ and $(e)]$ The $1 D$ line profiles of the 2D spectrum from Si (220) and Si (440). [(c) and (f)] The SASE spikes separated by $0.55 \mathrm{eV}$ and $0.15 \mathrm{eV}$ when using $\mathrm{Si}$ (220) and $\mathrm{Si}(440)$.
The 2D spectral images using the Si (333) and C (220) crystal reflection in the single-bunch mode are shown in Figs. 11(a) and 11(d), respectively. Figures 11 (b) and 11 (e) show the spectral projections of the $2 \mathrm{D}$ spectrograms normal to the dispersion direction. The $\mathrm{Si}$ (333) reflection provides high resolution with a minimum spike separation of $0.15 \mathrm{eV}$, as shown in Fig. 11(c), and C (220) yields a spike separation of $0.25 \mathrm{eV}$, as shown in the inserted Fig. 11(f). Calibration of the energy dispersion axis of the detector was performed by first recording the spectra with HIREX at one machine and detector setting, then shifted the central photon energy by a known amount by changing the undulator settings, and then recorded again HIREX spectra (without changing crystal and detector settings). The absolute photon energy is known from the electron beam energy and undulator settings not better than to about $\pm 50 \mathrm{eV}$ at a central photon energy of $9.3 \mathrm{keV}$, which is due to the measurement uncertainty of the absolute electron beam energy. However, any relative photon energy shift is known to be better than $1 \mathrm{eV}$, largely sufficient to determine the SASE bandwidth and to calibrate the HIREX detector.

The 1D spectral images from using C (220) and Si (220) in the multi-bunch mode are shown in Figs. 12 and 13. The data show all 20 consecutive spectra within one train. Figures 12(a) and 13(a) shows the X-ray Gas Monitor (XGM) ${ }^{38,39}$ pulse energies $\sim 2 \mathrm{~mJ}$ with 20 pulses in a train. Figure 12(b) shows the consecutive 20 spectra
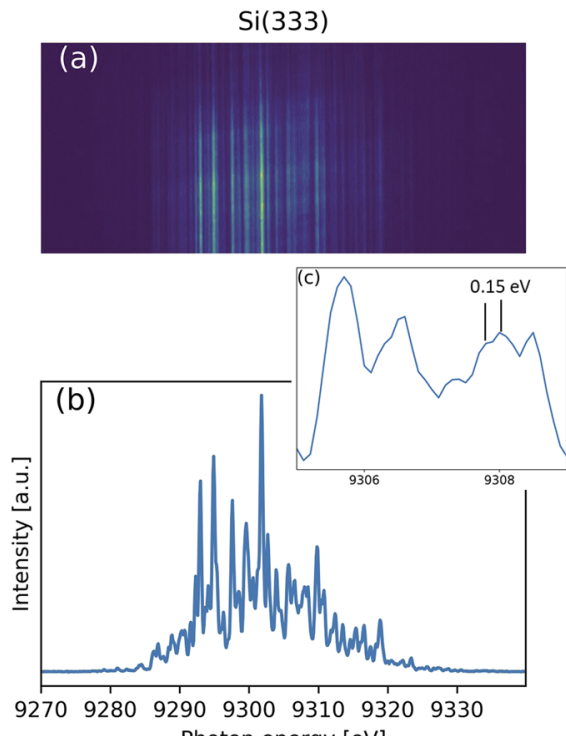

Photon energy [eV]
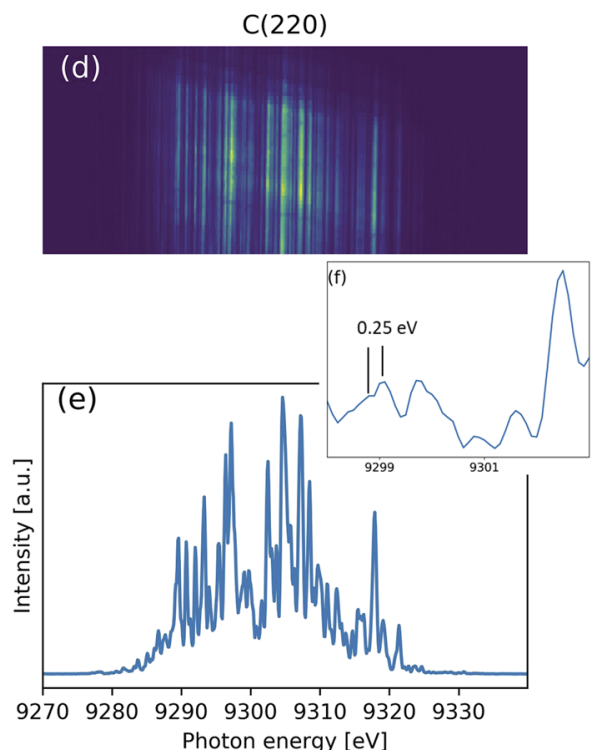

FIG. 11. Single-shot spectra using the $\mathrm{Si}$ (333) reflection with a $75 \mathrm{~mm}$ bending radius and from $C(220)$ reflection with a $125 \mathrm{~mm}$ bending radius at $9.3 \mathrm{keV}$. [(a) and (d)] The 2D spectrograms from the optical detector system. [(b) and (e)] The $1 \mathrm{D}$ line profiles of the $2 \mathrm{D}$ spectrum from Si (333) and C (220). [(c) and (f)] The SASE spikes separated by $0.15 \mathrm{eV}$ and $0.25 \mathrm{eV}$ when using $\mathrm{Si}(333)$ and $\mathrm{C}(220)$. 

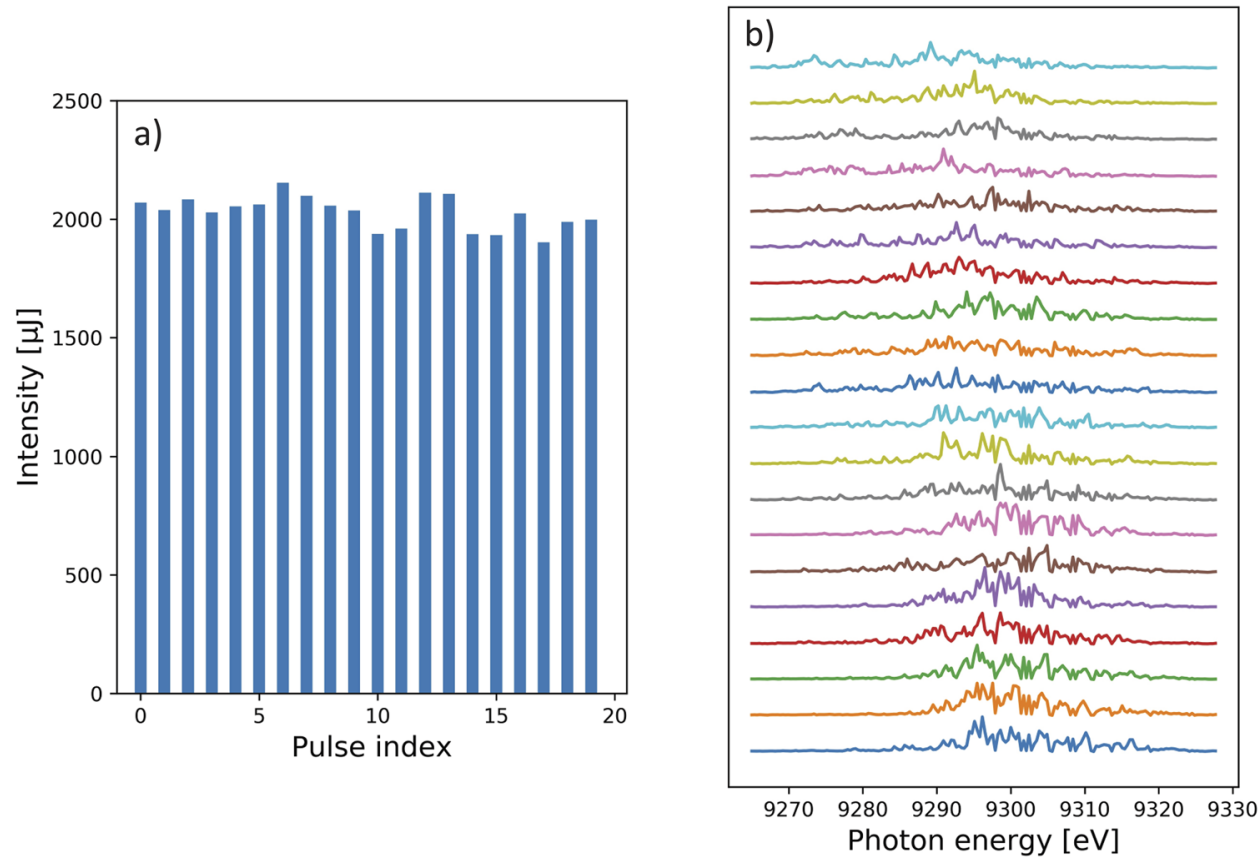

FIG. 12. (a) X-ray Gas Monitor (XGM) measurements showing $\sim 2 \mathrm{~mJ}$ pulse energy with intra-train pulse-resolution based on fast $A D C$ signals for 20 pulses within the train. (b) Single-shot spectra using the $C(220)$ reflection in the direct beam for 20 consecutive pulses within a train measured with the 1D GOTTHARD detector.

using the $\mathrm{C}(220)$ reflection recording with the GOTTHARD detector at a central photon energy of $9.3 \mathrm{keV}$, and Fig. 13(b) shows the consecutive 20 spectra using the $\mathrm{Si}(220)$ reflection with a bending radius of $100 \mathrm{~mm}$ in the first order diffracted beam from the grating with $200 \mathrm{~nm}$ pitch recorded by the GOTTHARD detector at a central photon energy of $9.3 \mathrm{keV}$. Each spectrum has different peak amplitudes at different places within the envelope of the SASE spectrum. Figure 14 shows the ensemble of single-shot spectra within a train using the Si (220) reflection. An ensemble of spectra of 600 shots is shown in light gray color, a randomly selected single-shot spectrum is shown in black color, and an ensemble average spectrum is shown in dark gray color.

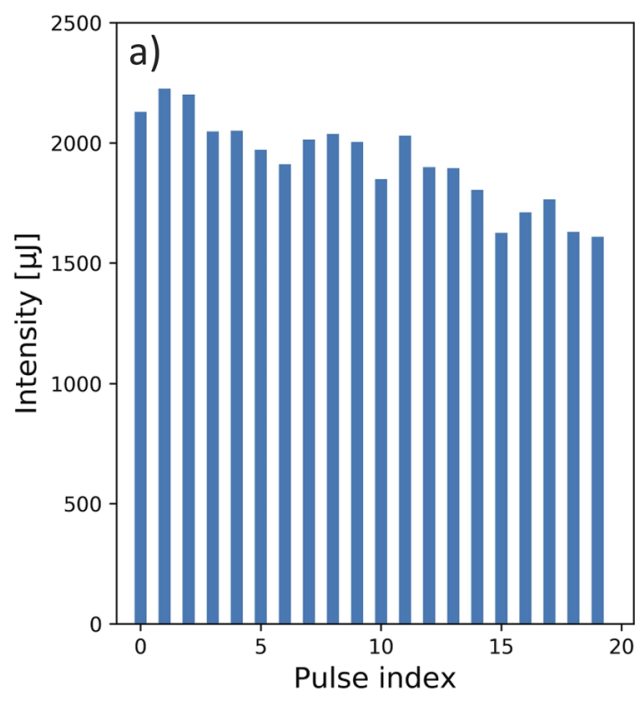

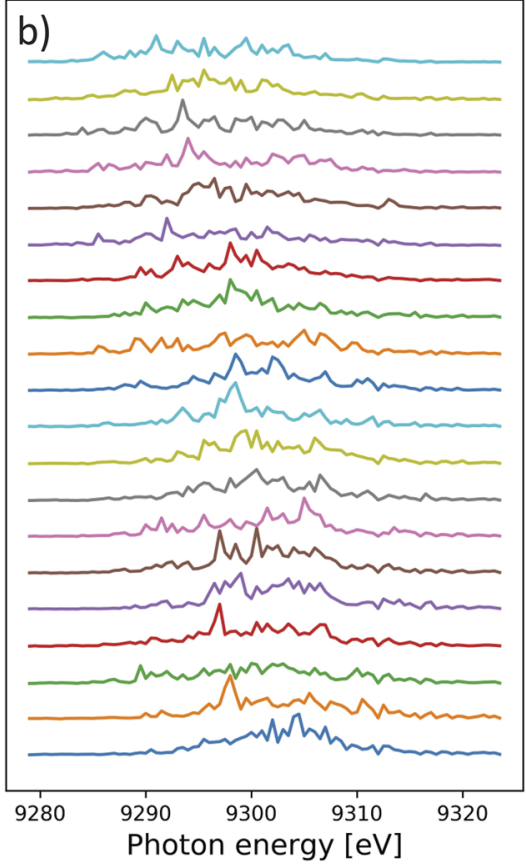

FIG. 13. (a) The X-ray Gas Monitor (XGM) measurements showing $\sim 2 \mathrm{~mJ}$ pulse energy with intra-train pulse resolution based on fast $A D C$ signals for 20 pulses within the train. (b) Singleshot spectra using the $\mathrm{Si}(220)$ reflection with a bending radius of $100 \mathrm{~mm}$ in the first order diffraction beam from the grating with $200 \mathrm{~nm}$ pitch for 20 consecutive pulses within a train measured with the 1D GOTTHARD detector. 


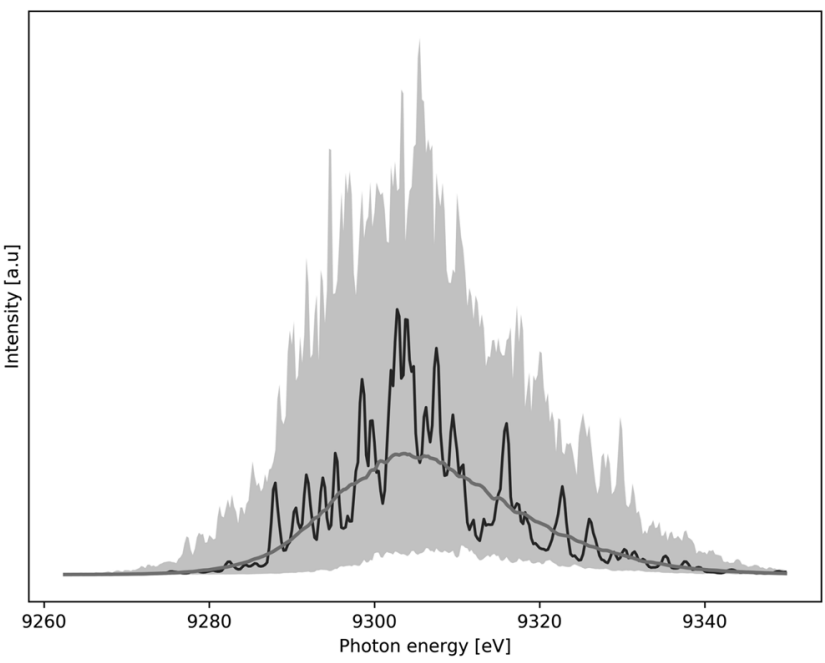

FIG. 14. An ensemble of 600-shot spectra obtained with the Si (220) crystal (light gray), a randomly picked single-shot spectrum (black line), and an ensemble average (dark gray line)

\section{CONCLUSION AND OUTLOOK}

The HIREX spectrometer can acquire single-shot spectra in the full EuXFEL photon energy range of $3 \mathrm{keV}-25 \mathrm{keV}$ by selecting different crystals. The entire SASE spectrum bandwidth at the central photon energy is recorded with a resolving power of 60000 to resolve individual spikes with $0.15 \mathrm{eV}$ separation using the $\mathrm{Si}$ 440 reflection. The measured SASE bandwidth is $25 \mathrm{eV}$ FWHM at $9.3 \mathrm{keV}$. Nearly all mechanical motions and absolute encoders were chosen to be outside the vacuum chamber to avoid potential contamination, as the HIREX spectrometer is close to the distribution mirror in the particle-free area beamline vacuum section in the XTD9 tunnel. Only the crystal rotation encoder is placed inside the vacuum system in order to achieve the highest accuracy of the angular positioning. Depending on the exact operating parameters of the machine, the best combination of crystal orientation and bending radius is selected to accommodate the photon energy, spectral range, and resolution. The required spectral range and resolution are obtained with silicon and diamond crystals with various reflections and bending radii. The high-repetition-rate GOTTHARD detector allows operating the HIREX spectrometer at $\mathrm{MHz}$ repetition rates. The currently installed GOTTHARD detector operates up to $\sim 1 \mathrm{MHz}$. At the end of this year, we will upgrade to a next-version GOTTHARD detector that operates up to $4.5 \mathrm{MHz}$ and will thus match the maximum EuXFEL repetition rate. We are planning to automate the HIREX spectrometer with an intuitive simple user control interface for instrument scientists and machine operators. Recently, we have installed another HIREX spectrometer in the SASE2 beamline, and commissioning of that spectrometer is in progress. It has already been used for self-seeding studies.

\section{ACKNOWLEDGMENTS}

We would like to thank P. Karvinen from Finnlitho Ltd., Finland, for discussions about the project as well as J. Rehanek,
P. Juranić, and C. David from the Paul-Scherrer Institute (PSI), Switzerland, and U. Wissemann from Axilon AG, Hürth, Germany. Diamond Materials GmbH, Freiburg, Germany, provided the freestanding diamond membranes. Norcada Inc., Canada, provided the thin silicon crystals. Y. Tong from Shanghai University of Technology, China, helped in the data analysis. S. Terentyev from TISNCM, Russia, provided the bent HPHT type IIa diamond crystal. All the authors would like to thank the members of the EuXFEL groups, X-ray Optics, Vacuum, Controls, Electronic and Electrical Engineering (EEE), and IT and Data Management (ITDM), as well as the DESY alignment group and the DESY MXL group, for their support. We would like to thank all instrument scientists of FXE and SPB/SFX. We would like to thank K. Ament for proofreading this manuscript, and we gratefully acknowledge the EuXFEL Management Board for supporting and funding this project.

\section{DATA AVAILABALITY}

The data that support the findings of this study are available from the corresponding author upon reasonable request.

\section{REFERENCES}

${ }^{1}$ M. Altarelli, R. Brinkmann, M. Chergui, W. Decking, B. Dobson, S. Düsterer, G. Grubel, W. Graeff, H. Graafsma, J. Hajdu, J. Marangos, J. Pflüger, H. Redlin, D. Riley, I. Robinson, J. Rossbach, A. Schwarz, K. Tiedtke, T. Tschentscher, I. Vartaniants, H. Wabnitz, H. Weise, R. Wichmann, K. Witte, A. Wolf, M. Wulff, and M. Yurkov, "The European X-ray free-electron laser: Technical design report," DESY Report No. 2006-097, DESY, Hamburg, Germany, 2006.

${ }^{2}$ M. Altarelli, Nucl. Instrum. Methods Phys. Res., Sect. B 269, 2845 (2011).

${ }^{3}$ T. Tschentscher, C. Bressler, J. Grünert, A. Madsen, A. Mancuso, M. Meyer, A. Scherz, H. Sinn, and U. Zastrau, Appl. Sci. 7, 592 (2017).

${ }^{4}$ E. L. Saldin, E. V. Schneidmiller, and M. V. Yurkov, The Physics of Free-Electron Lasers, 1st ed. (Springer, Berlin Germany, 1999).

${ }^{5}$ C. Pellegrini, A. Marinelli, and S. Reiche, Rev. Mod. Phys. 88, 015006 (2016).

${ }^{6}$ A. M. Kondratenko and E. L. Saldin, Part. Accel. 10, 207 (1980).

${ }^{7}$ R. Bonifacio, C. Pellegrini, and L. M. Narducci, Opt. Commun. 50, 373 (1984).

${ }^{8}$ H. J. Kirkwood, R. Letrun, T. Tanikawa, J. Liu, M. Nakatsutsumi, M. Emons, T. Jezynski, G. Palmer, M. Lederer, R. Bean, J. Buck, S. Di Dio Cafisio, R. Graceffa, J. Grünert, S. Göde, H. Höppner, Y. Kim, Z. Konopkova, G. Mills, M. Makita, A. Pelka, T. R. Preston, M. Sikorski, C. M. S. Takem, K. Giewekemeyer, M. Chollet, P. Vagovic, H. N. Chapman, A. P. Mancuso, and T. Sato, Opt. Lett. 44, 1650 (2019).

${ }^{9}$ T. sato, R. Letrun, H. J. Kirkwood, J. Liu, P. Vagovič, G. Mills, Y. Kim, C. M. S. Takem, M. Planas, M. Emons, T. Jezynski, G. Palmer, M. Lederer, S. Schulz, J. Mueller, H. Schlarb, A. Silenzi, G. Giovanetti, A. Parenti, M. Bergemann, T. Michelat, J. Szuba, J. Grünert, H. N. Chapman, and A. P. Mancuso, Optica 7, 716-717 (2020).

${ }^{10}$ P. Emma, R. Akre, J. Arthur, R. Bionta, C. Bostedt, J. Bozek, A. Brachmann, P. Bucksbaum, R. Coffee, F.-J. Decker, Y. Ding, D. Dowell, S. Edstrom, A. Fisher, J. Frisch, S. Gilevich, J. Hastings, G. Hays, P. Hering, Z. Huang, R. Iverson, H. Loos, M. Messerschmidt, A. Miahnahri, S. Moeller, H.-D. Nuhn, G. Pile, D. Ratner, J. Rzepiela, D. Schultz, T. Smith, P. Stefan, H. Tompkins, J. Turner, J. Welch, W. White, J. Wu, G. Yocky, and J. Galayda, Nat. Photonics 4, 641 (2010).

${ }^{11}$ T. Ishikawa, H. Aoyagi, T. Asaka, Y. Asano, N. Azumi, T. Bizen, H. Ego, K. Fukami, T. Fukui, Y. Furukawa et al., Nat. Photonics 6, 540 (2012).

${ }^{12}$ I. S. Ko, H. S. Kang, H. Heo, C. Kim, G. Kim, C. K. Min, H. Yang, S. Y. Baek, H. J. Choi, G. Mun et al., Appl. Sci. 7, 479 (2017).

${ }^{13}$ B. D. Patterson, R. Abela, H.-H. Braun, U. Flechsig, R. Ganter, Y. Kim, E. Kirk, A. Oppelt, M. Pedrozzi, S. Reiche, L. Rivkin, T. Schmidt, B. Schmitt, V. N. Strocov, S. Tsujino, and A. F. Wrulich, New J. Phys. 12, 035012 (2010).

${ }^{14}$ W. Decking et al., Nat. Photonics 14, 391 (2020). 
${ }^{15}$ J. Grünert, "Framework for x-ray photon diagnostics at the European XFEL," XFEL.EU TR-2012-003, 2012.

${ }^{16}$ J. Grünert, M. P. Carbonell, F. Dietrich, T. Falk, W. Freund, A. Koch, N. Kujala, J. Laksman, J. Liu, T. Maltezopoulos, K. Tiedtke, U. F. Jastrow, A. Sorokin, E. Syresin, A. Grebentsov, and O. Brovko, J. Synchrotron Radiat. 26, 1422 (2019).

${ }^{17}$ J. Grünert, A. Koch, N. Kujala, W. Freund, M. Planas, F. Dietrich, J. Buck, J. Liu, H. Sinn, M. Dommach, and S. Molodtsov, in Proceedings of the 37th International Free Electron Laser Conference (FEL 2015), WED03, 23-28 August 2015, Daejeon, South Korea, 2015.

${ }^{18}$ J. Grünert, M. P. Carbonell, F. Dietrich, W. Freund, A. Koch, N. Kujala, J. Laksman, J. Liu, and T. Maltezopoulos, AIP Conf. Proc. 2054, 030014 (2019).

${ }^{19}$ E. L. Saldin, E. A. Schneidmiller, and M. V. Yurkov, Nucl. Instrum. Methods Phys. Res., Sect. A 407, 291 (1998).

${ }^{20}$ Y. Inubushi, I. Inoue, J. Kim, A. Nishihara, S. Matsuyama, H. Yumoto, T. Koyama, K. Tono, H. Ohashi, K. Yamauchi, and M. Yabashi, Appl. Sci. 7, 584 (2017).

${ }^{21}$ M. Yabashi, J. B. Hastings, M. S. Zolotorev, H. Minura, H. Yumoto, S. Matsuyama, K. Yamauchi, and T. Ishikawa, Phys. Rev. Lett. 97, 084802 (2006).

${ }^{22}$ D. Rich, D. Zhu, J. Turner, D. Zhang, B. Hill, and Y. Feng, J. Synchrotron Radiat. 23, 3 (2016).

${ }^{23}$ J. Rehanek, M. Makita, P. Wiegand, P. Heimgartner, C. Pradervand, G. Seniutinas, U. Flechsig, V. Thominet, C. W. Schneider, A. R. Fernandez, C. David, L. Patthey, and P. Juranić, J. Instrum. 12, P05024 (2017).

${ }^{24}$ J. Rehanek, N. Kujala, J. Grünert, “Technical design report,” XFEL.EU Alfresco Doc-ID:13779761, 2014.

${ }^{25}$ J. Rehanek, F. Schäfers, A. Erko, W. Freund, J. Grünert, C. Ozkan, and S. Molodtsov, Proc. SPIE 8141, 814109 (2011).

${ }^{26}$ D. Zhu, M. Cammarata, J. M. Feldkamp, D. M. Fritz, J. B. Hastings, S. Lee, H. T. Lemke, A. Robert, J. L. Turner, and Y. Feng, Appl. Phys. Lett. 101, 034103 (2012).

${ }^{27}$ C. David, S. Gorelick, S. Rutishauser, J. Krzywinski, J. Vila-Comamala, V. A. Guzenko, O. Bunk, E. Färm, M. Ritala, M. Cammarata, D. M. Fritz, R. Barrett, L. Samoylova, J. Grünert, and H. Sinn, Sci. Rep. 1, 57 (2011).
${ }^{28} \mathrm{P}$. Karvinen, S. Rutishauser, A. Mozzanica, D. Greiffenberg, P. N. Juranić, A. Menzel, A. Lutman, J. Krzywinski, D. M. Fritz, H. T. Lemke, M. Cammarata, and C. David, Opt. Lett. 37, 5073 (2012).

${ }^{29}$ M. Makita, P. Karvinen, D. Zhu, P. N. Juranic, J. Grünert, S. Cartier, J. H. Jungmann-Smith, H. T. Lemke, A. Mozzanica, S. Nelson, L. Patthey, M. Sikorski, S. Song, Y. Feng, and C. David, Optica 2, 912 (2015).

${ }^{30}$ M. Makita, P. Karvinen, V. A. Guzenko, N. Kujala, P. Vagovic, and C. David, Microelectron. Eng. 176, 75 (2017).

${ }^{31}$ N. Kujala, M. Makita, J. Liu, A. Zozulya, M. Sprung, C. David, and J. Grünert, J. Synchrotron Radiat. 26, 708 (2019).

${ }^{32}$ H. Sinn, J. Gaudin, L. Samoylova, A. Trapp, and G. Galasso, "Conceptual design report: X-ray optics and beam transport," XFEL.EU, TR-2011-002, 2011.

${ }^{33}$ S. N. Polyakov, V. N. Denisov, N. V. Kuzmin, M. S. Kuznetsov, S. Y. Martyushov, S. A. Nosukhin, S. A. Terentiev, and V. D. Blank, Diamond Relat. Mater. 20, 726-728 (2011).

${ }^{34}$ U. Boesenberg, L. Samoylova, T. Roth, D. Zhu, S. Terentyev, M. Vannoni, Y. Feng, T. B. van Driel, S. Song, V. Blank, H. Sinn, A. Robert, and A. Madsen, Opt. Express 25, 2852 (2017).

${ }^{35}$ L. Samoylova, U. Boesenberg, A. Chumakov, V. Kaganer, I. Petrov, T. Roth, R. Rüffer, H. Sinn, S. Terentyev, and A. Madsen, J. Synchrotron Radiat. 26, 1069 (2019).

${ }^{36}$ S. Terentyev, V. Blank, T. Kolodziej, and Y. Shvyd'ko, Rev. Sci. Instrum. 87, 125117 (2016).

${ }^{37}$ A. Mozzanica, A. Bergamaschi, R. Dinapoli, H. Graafsma, D. Greiffenberg, B. Henrich, I. Johnson, M. Lohmann, R. Valeria, B. Schmitt, and S. Xintian, J. Instrum. 7, C01019 (2012).

${ }^{38}$ T. Maltezopoulos, F. Dietrich, W. Freund, U. F. Jastrow, A. Koch, J. Laksman, J. Liu, M. Planas, A. A. Sorokin, K. Tiedtke, and J. Grünert, J. Synchrotron Radiat. 26, 1045 (2019)

${ }^{39}$ A. A. Sorokin, Y. Bican, S. Bonfigt, M. Brachmanski, M. Braune, U. F. Jastrow, A. Gottwald, H. Kaser, M. Richter, and K. Tiedtke, J. Synchrotron Radiat. 26, 1092 (2019). 